\title{
Triplet-triplet annihilation photon-upconversion in hydrophilic media with biorelevant cholesteryl triplet energy acceptors
}

\section{Yun, Young Ju}

2021-09-01

Yun , Y J , Isokuortti , J , Laaksonen , T , Durandin , N \& Ayitou , A J L 2021 , ' Triplet-triplet annihilation photon-upconversion in hydrophilic media with biorelevant cholesteryl triplet energy acceptors ' , Journal of Photochemistry and Photobiology A: Chemistry, vol. 418 , 113412 . https://doi.org/10.1016/j.jphotochem.2021.113412

http://hdl.handle.net/10138/336865

https://doi.org/10.1016/j.jphotochem.2021.113412

unspecified

submittedVersion

Downloaded from Helda, University of Helsinki institutional repository.

This is an electronic reprint of the original article.

This reprint may differ from the original in pagination and typographic detail.

Please cite the original version. 
Graphical Abstract

Graphical Abstract
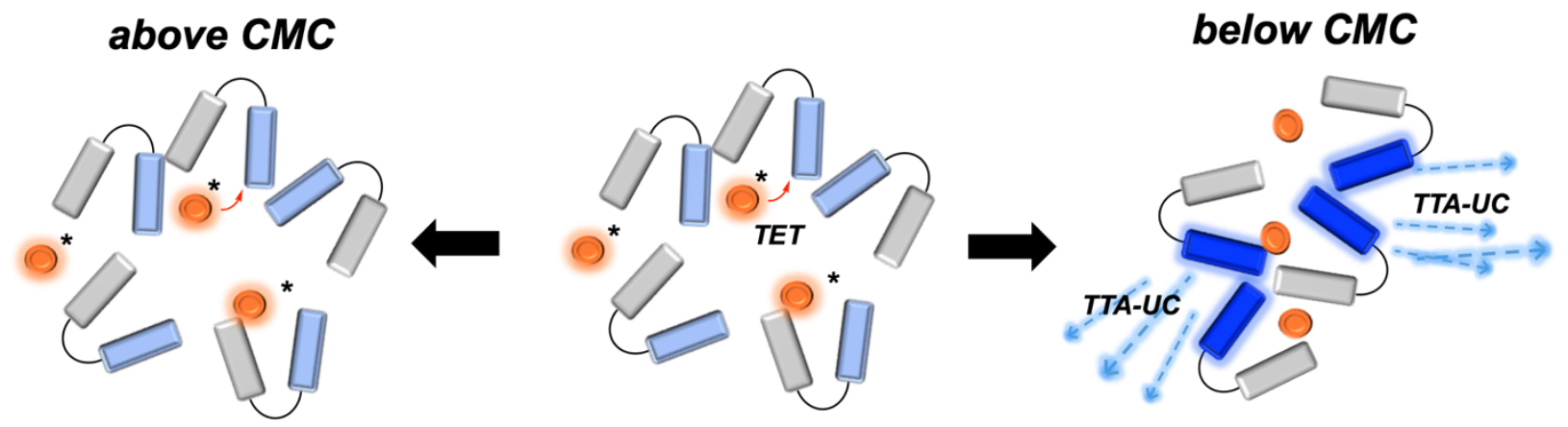

Inefficient TET process 


\section{Triplet-Triplet Annihilation Photon-Upconversion in Hydrophilic Media with Biorelevant Cholesteryl Triplet Energy Acceptors}

Young Ju Yun ${ }^{\ddagger}, \#$, Jussi Isokuortti ${ }^{\diamond, \#}$, Timo Laaksonen ${ }^{\diamond, \dagger}$, Nikita Durandin ${ }^{\diamond}$ and A. Jean-Luc Ayitou ${ }^{\ddagger *}$

$\$$ Department of Chemistry, Illinois Institute of Technology, Chicago, IL 60616, United States.

${ }^{\diamond}$ Engineering and Natural Sciences, Tampere University, Korkeakoulunkatu 8, 33720 Tampere, Finland.

$\dagger$ Division of Pharmaceutical Biosciences, Faculty of Pharmacy, University of Helsinki, Viikinkaari 5 E, 00014 Helsinki, Finland.

\# These authors have contributed equally to this work

*Corresponding author E-mail

aayitou@iit.edu 
ABSTRACT: We report two new biorelevant cholesteryl-based triplet energy acceptors, derivatives of DPA or 9,10-diphenylanthracene (C-DPA and $\left.\mathbf{C}_{2}-\mathbf{D P A}\right)$. Using two different triplet sensitizers: QDN $\left(\mathrm{E}_{\mathrm{T}} \approx 1.77 \mathrm{eV}\right)$ and $\mathbf{P d T P P}\left(\mathrm{E}_{\mathrm{T}} \approx 1.78 \mathrm{eV}\right)$, we were able to achieve both endothermic (with QDN) and exothermic (with PdTPP) triplet sensitization of DPA, C-DPA and C2-DPA in hydrophilic PEG200 media. While the maximum rate of triplet energy transfer (TET) was achieved with PdTPP and DPA $\left(k_{\mathrm{TET}}=4.7 \times 10^{7} \mathrm{M}^{-1} \mathrm{~s}^{-1}\right)$, for the cholesteryl-based acceptors, we found that the kinetic of the TET process was dependent upon the concentration of the acceptor. For PdTPP/C-DPA pair, the rate of the dynamic triplet energy quenching was $k_{\mathrm{TET}}=1.9 \times 10^{7}$ $\mathrm{M}^{-1} \mathrm{~s}^{-1}$; however, at higher concentrations of the quencher, the system reached a stationary state due to formation of self-assembled sub-domains of C-DPA that likely slowed the TET process. It was also found that this aggregation of C-DPA in PEG200 led to a 3.5 folds increase in the $\mathrm{I}_{\text {th }}$ compared to $133 \mathrm{~mW} \mathrm{~cm}{ }^{2}$ for DPA. Subsequently, we estimated the $\Phi_{U C}$ for these donor/acceptor pairs: QDN/DPA, PdTPP/DPA, and PdTPP/C-DPA. With respect to the estimated $\mathrm{I}_{\text {th }}$, we found that the uncorrected (for inner filter effect) quantum yields of TTA-UC were $2 \leq \Phi_{U C} \leq 12 \%$. 


\section{INTRODUCTION:}

Triplet-triplet annihilation photon-upconversion (TTA-UC) is an elegant photophysical process that allows to amplify photon energy for applications at the interface of solar energy conversion, optoelectronic science, and bio-imaging. ${ }^{1-7}$ It has been well-documented that TTA-UC is a bi-molecular process, where the triplet energy donor/sensitizer absorbs low-energy light and the triplet energy acceptor emits higher-energy photons. ${ }^{8,9}$ Under this condition, TTA-UC has been shown to be a promising process vis-à-vis bio-imaging applications, where low-energy light is amplified and the resulting emission could be tailored for in vivo photo-excitation of biological markers ${ }^{10}$ or even achieve photochemical/biochemical transformations. ${ }^{11,12}$ In this scenario, TTAUC chromophoric systems can be systematically functionalized with biocompatible/biorelevant groups such as cholesteryl in order to facilitate the integration of the modified donor/acceptor dyes into biological environments. In addition, the derivatized dyes could be used to study the underlying photo-kinetics and efficiency of the processes involved in TTA-UC in lipids and membranes. To study TTA-UC in lipids and membranes, it's also important to mimic the relevant conditions viz. viscosity, hydro-philicity/phobicity, and polarity of biological environments. A recent strategy in this regards proposed to achieve the TTA-UC processes in PEG200, which is a hydrophilic polymeric liquid that mimics cell membrane structures and intracellular conditions. ${ }^{13-}$

${ }^{16}$ With this in mind, we are hereby reporting two new cholesteryl-based 9,10-diphenylanthracene (DPA) derivatives (C-DPA and $\mathbf{C}_{\mathbf{2}}$-DPA) (Scheme 1) which we used as triplet energy acceptors for TTA-UC in PEG200. The present study also aimed at deciphering the effect of the biorelevant cholesteryl group on donor.••acceptor interactions and the resulting TTA-UC process. 


\section{A) Donors / Sensitizers}

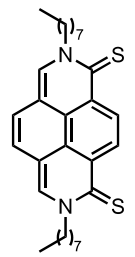

QDN

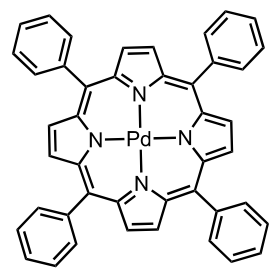

PdTPP

B) Acceptors / Annihilators<smiles>c1ccc(-c2c3ccccc3c(-c3ccccc3)c3ccccc23)cc1</smiles>

DPA

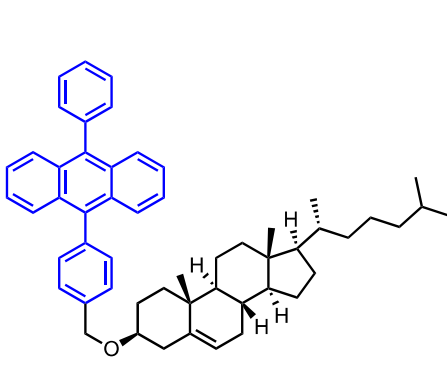

C-DPA

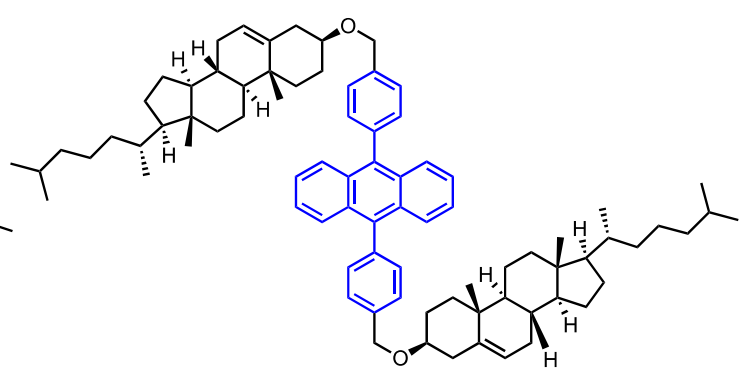

$\mathrm{C}_{2}-\mathrm{DPA}$

Scheme 1. Chemical structures of sensitizers (QDN and PdTPP) and annihilators (DPA, C-DPA, and $\mathbf{C}_{2}-\mathbf{D P A}$ ).

Recently, a new family of triplet sensitizers: quinoidal naphthyl thioamides (QDN) (Scheme 1) were reported by our group. ${ }^{17}$ QDN are structurally compatible with over-the-counter acceptors/fluorescent polyaromatic hydrocarbons such as perylene ${ }^{18,19}$ and 9,10diphenylanthracene (DPA) which are routinely employed for TTA-UC processes. We had previously established that the triplet energy of QDN $\left(\mathrm{E}_{\mathrm{T}} \approx 1.77 \mathrm{eV}\right.$, in $77 \mathrm{~K}$ EtOH:DCM glassy matrix $)$ is suitable for an exothermic energy transfer to perylene $\left(\mathrm{E}_{\mathrm{T}} \approx 1.53 \mathrm{eV}\right)$; however, it was unexpected that $\mathbf{Q D N}$ could be used to sensitize DPA $\left(\mathrm{E}_{\mathrm{T}} \approx 1.78 \mathrm{eV}\right)^{20}$ in deoxygenated PEG200, since its measured $\mathrm{E}_{\mathrm{T}}$ value at room temperature in that medium was estimated at $1.67 \mathrm{eV}$, far below the triplet energy of DPA. It's worth noting that, in order to maintain the deoxygenated state 
of all of our photo-samples, $30 \mathrm{mM}$ of oleic acid was added to the PEG200 material (oleic acid was used as oxygen scavenger). Triplet sensitization study of DPA using QDN and/or another conventional sensitizer 5,10,15,20-TetraPhenylPorphyrinato Palladium(II) (PdTPP) with $\mathrm{E}_{\mathrm{T}} \approx$ $1.78 \mathrm{eV}^{20}$ revealed that both endothermic $\mathbf{Q D N} \rightarrow \mathbf{D P A}$ and exothermic PdTTP $\rightarrow$ DPATET could be achieved in biorelevant PEG200 media. Armed with these exciting findings, we furthered the TTA-UC studies with the new cholesteryl-based triplet energy acceptors C-DPA and $\mathbf{C}_{2}$-DPA. Expectedly, the cholesteryl unit would not only enhance interactions of the hydrophobic donor••acceptor in the hydrophilic PEG200 medium, but also induce the formation of selfassembled sub-domains at higher concentrations of the donor/acceptor chromophoric systems.

\section{RESULTS \& DISCUSSION:}

The synthetic procedures for all precursors of C-DPA and $\mathbf{C}_{2}-\mathbf{D P A}$ are extensively described in the Supporting Information (Scheme S1). C-DPA and $\mathbf{C}_{2}$-DPA were synthesized following Suzuki coupling reaction conditions, where cholesteryl 4-bromobenzyl ether ( $\mathbf{B r}-\mathbf{P h C H} \mathbf{H}_{2}-\mathbf{O}-$

Chol) was reacted with 10-phenyl-9-anthracene boronic acid for C-DPA and 9,10Anthracenediboronic acid bis(pinacol) ester for C2-DPA (Supporting Information, Scheme S1S4). C-DPA and C2-DPA were successfully characterized by NMR (Supporting Information, Figure S1-S8), thermogravimetry (Supporting Information, Figure S12), and single-crystal XRD (Supporting Information, Figure S13). 

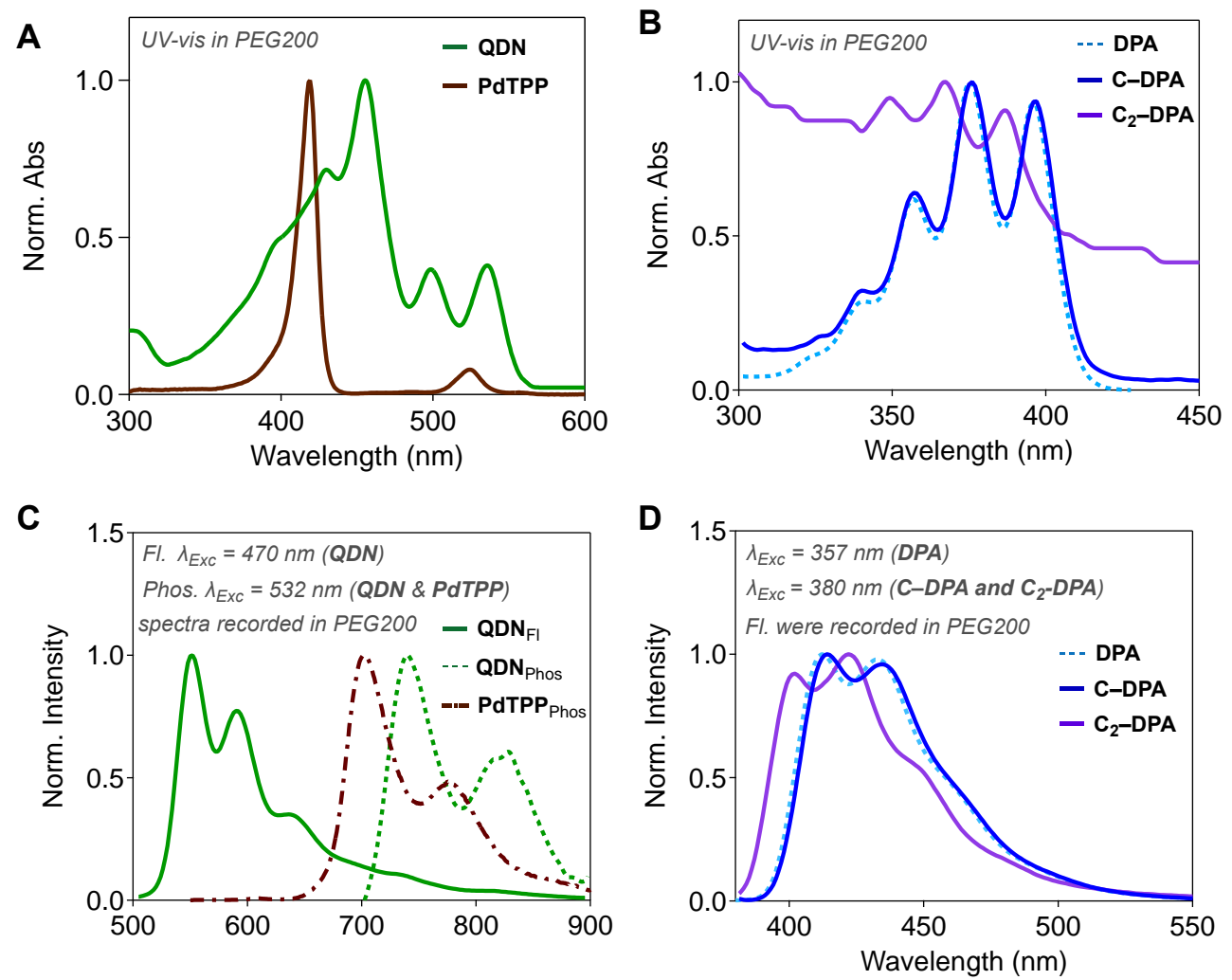

Figure 1. UV-vis profiles of (A) QDN and PdTPP and (B) DPA, C-DPA, and C $_{2}-$ DPA in PEG200. (C) Emission profiles of QDN and PdTPP and (D) DPA, C-DPA, and C2-DPA recorded in PEG200 with O.D. $=0.2$; at the excitation wavelength $532 \mathrm{~nm}$ for QDN and PdTPP, $357 \mathrm{~nm}$ for DPA, $380 \mathrm{~nm}$ for C-DPA and $\mathbf{C}_{2}$-DPA.

UV-vis absorption profiles of C-DPA and $\mathbf{C}_{2}$-DPA, recorded in PEG200, revealed the same transitions features that are seen in the spectrum of parent DPA (Figure 1B). The absorption features of C-DPA was precisely the same as parent DPA, which is not surprising. However, the spectrum of $\mathbf{C}_{2}$-DPA exhibits a slight hypsochromic-shift compared to C-PDA or DPA. The positive offset in the spectrum of $\mathbf{C}_{2}-\mathbf{D P A}$ is suggestive of self- or H-aggregation, which could be problematic for an efficient donor*aacceptor interaction in PEG200. Importantly the positive spectral shift was not observed in the absorption spectrum of $\mathbf{C}_{2}-\mathbf{D P A}$ in DCM solution (Supporting information, Figure S11). Similarly, the steady-state emission profiles of C-DPA and 
$\mathbf{C}_{2}$-DPA depict the typical vibronic bands of DPA with no apparent/trivial spectral shift. With this result, we ascertain that the cholesteryl unit has little impact on the optoelectronic transitions of the new acceptors (Figure 1D). Nevertheless, the emission spectra recorded in PEG200 are slightly red-shifted presumably due to aggregation or in the hydrophilic PEG200 medium. (Supporting information, Figure S9-S11).
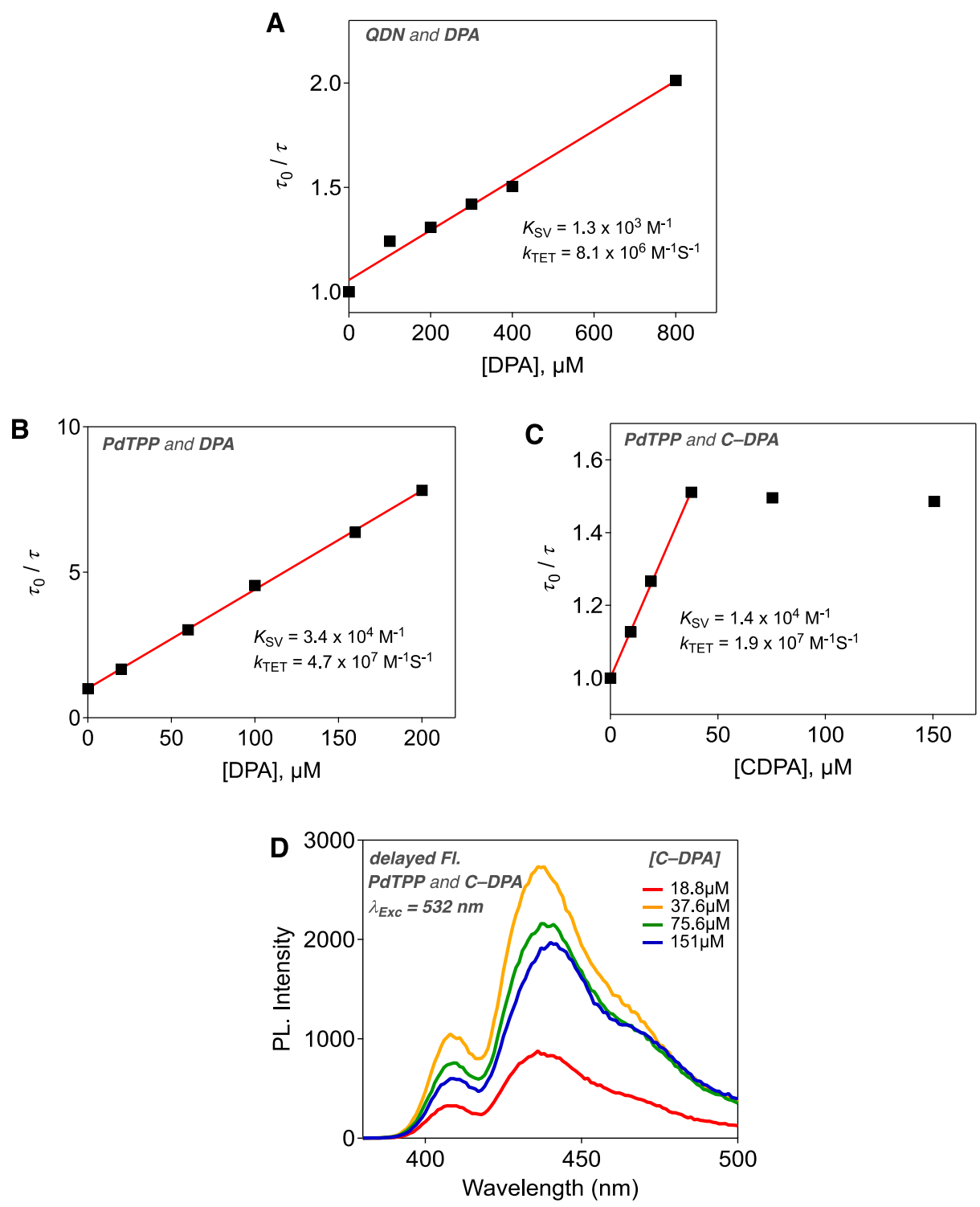

Figure 2. Stern-Volmer plots of (A) QDN and DPA (B) PdTPP and DPA (C) PdTPP and CDPA in PEG200 with the resulting Stern-Volmer constants $\left(K_{\text {SV }}\right)$ and TET rate constants $\left(k_{\text {TET }}\right)$. (D) Intensity-dependent delayed emission of C-DPA in deoxygenated PEG200 using PdTPP as 
the sensitizer and excitation wavelength of $532 \mathrm{~nm}$. Note: The phosphorescence samples were recorded in deoxygenated PEG200 in the presence of $30 \mathrm{mM}$ of oleic acid.

Next, we performed Stern-Volmer (S-V) quenching experiments, using conventional triplet energy acceptors e.g. DPA, to establish the best sensitizer (QDN vs. PdTPP) for efficient TET with both $\mathbf{C}-\mathbf{D P A}$ and $\mathbf{C}_{2}$-DPA. The quenching experiment was monitored by recording the phosphorescence of the two sensitizers in the presence of the acceptor/annihilator(s) with varying concentrations. The quenching data was analyzed following equations (1) and (2). ${ }^{21}$

$K_{S V}=K_{q} \tau_{0}$.

Where $K_{q}$ is the quenching rate constant, $\tau_{0}$ is the intrinsic phosphorescence lifetime of the sensitizer, and $K_{S V}$ is the Stern-Volmer constant.

For dynamic quenching mechanism, ${ }^{22}$ we presumed that

$\frac{\tau_{0}}{\tau}-1=K_{q} \tau_{0}[Q]$

Where $\tau$ is the phosphorescence lifetime of the sensitizer in the presence of the acceptor/annihilator(s), and $[\mathrm{Q}]$ is the concentration of acceptor/annihilator(s).

Figure 2 shows the $\mathrm{S}-\mathrm{V}$ plots with respect to the concentration of annihilator/quencher. The slope of the S-V plot is determined as $K_{S V}=K_{q} \tau_{0}$ (Eq. 1). Next, the rate of triplet energy transfer $\left(k_{T E T}\right)$ was computed from the following equation:

$k_{T E T}=\frac{K_{S V}}{\tau_{0}}$ 
$\tau_{0}$ for QDN was $160 \mu \mathrm{s}$, and that of PdTPP was $720 \mu \mathrm{s}$ (both values were measured in in PEG200). We then established that for DPA, the TET rate constant $k_{\mathrm{TET}}=8.1 \times 10^{6} \mathrm{M}^{-1} \mathrm{~s}^{-1}$ for QDN and $k_{\mathrm{TET}}=4.7 \times 10^{7} \mathrm{M}^{-1} \mathrm{~s}^{-1}$ for PdTPP (Figures 2A \& 2B). As one can see, the $k_{T E T}$ for QDN $\rightarrow$ DPA revealed an endothermic energy transfer with $\Delta \mathrm{E}_{\mathrm{T}}=-0.046 \mathrm{eV}$, whereas $\mathbf{P d T P P} \rightarrow$ DPA TET with $\Delta \mathrm{E}_{\mathrm{T}}=-0.01 \mathrm{eV}$ is an exothermic process (assuming a rate of diffusion $k_{\text {diff }}=5 \times 10^{7}$ in PEG200). ${ }^{13,14}$ These values indicate that for an efficient TET, the later sensitizer is the preferred triplet energy donor for both $\mathbf{C}-$ DPA and $\mathbf{C}_{2}-\mathbf{D P A}$. The $\mathrm{S}-\mathrm{V}$ quenching experiments are summarized in Figure 2. While the phosphorescence quenching with DPA depicts the expected dynamic quenching behavior, the quenching study with C-DPA reached a stationary state after certain threshold concentration of the quencher. For example, as shown in Figure 2C, at low/medium concentration of C-DPA, the $k_{T E T}=1.9 \times 10^{7} \mathrm{M}^{-1} \mathrm{~s}^{-1}$ for PdTPP $\rightarrow \mathbf{C}-\mathbf{D P A}$ in the dynamic regime was three times smaller than that of PdTPP $\rightarrow$ DPA suggesting hindered intermolecular interactions/collisions due to the presence of the cholesteryl moieties that may be shielding the DPA core. In this picture, we also hypothesized that at micellar concentration of 39 $\mu \mathrm{M}$, there is a possibility of back DPA $\rightarrow$ PdTPP energy transfer via the FRET mechanism. 


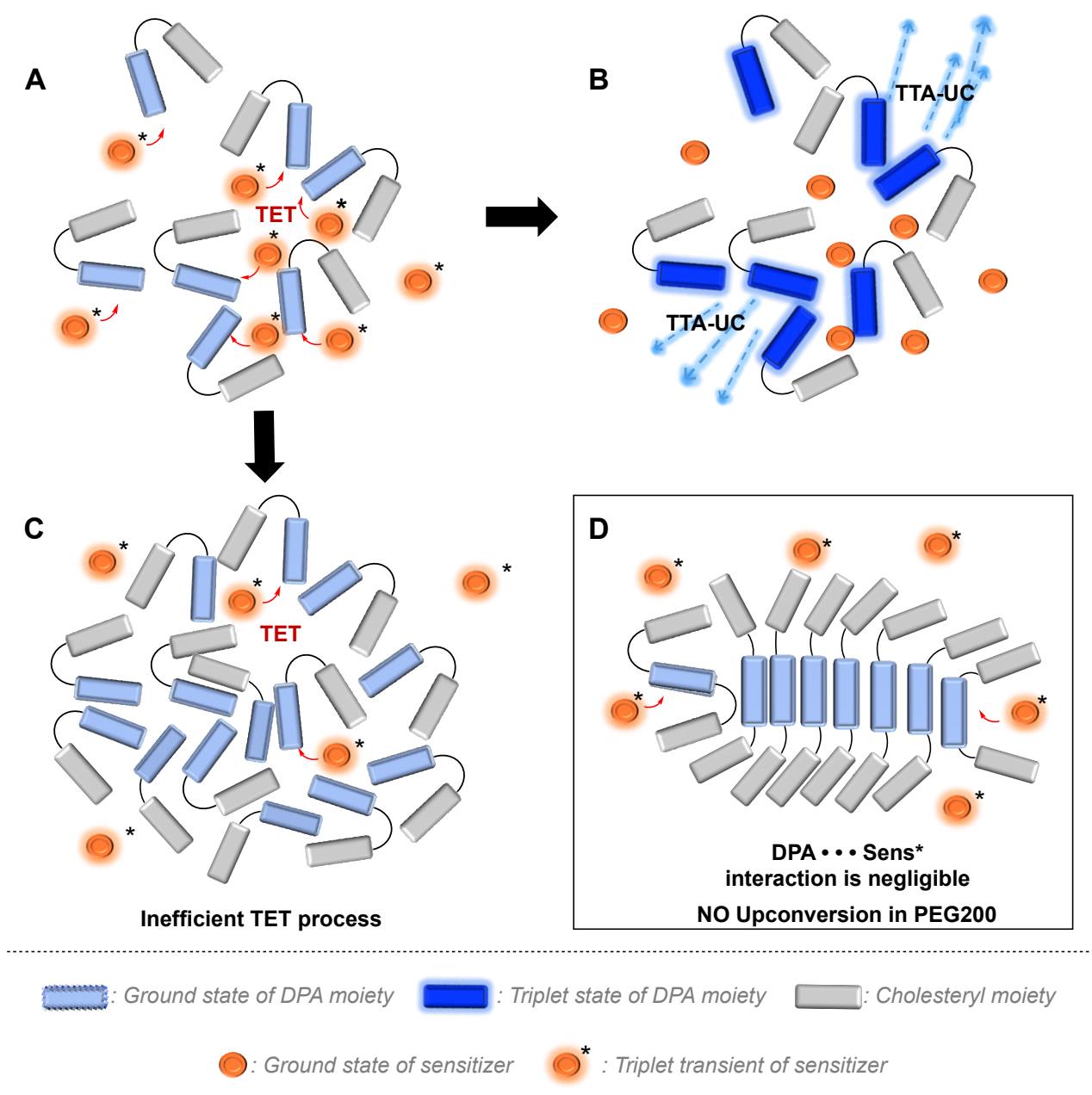

Figure 3. A cartoon depicting the energy flow and mechanism of TTA-UC. Proposed aggregation features of (A) C-DPA with low \& medium concentration, (C) C-DPA with high concentration, and (D) C2 - DPA in PEG200. (B) Proposed TTA-UC of C-DPA. The sensitizer represents PdTPP or QDN.

From the $\mathrm{S}-\mathrm{V}$ quenching studies, we deduced that the maximum TET and possibly upconversion process could be achieved at $[\mathbf{C}-\mathbf{D P A}]=39 \mu \mathrm{M}$ with $34 \%$ quenching efficiency rate (without any aggregation of the acceptor). We attributed the plateau of the S-V curved beyond [CDPA $]=39 \mu \mathrm{M}$ to the aggregation of $\mathbf{C}-\mathbf{D P A}$ units to form self-assembled sub-domains or phaseseparation of C-DPA, which was visible to the naked eye, indicating that the critical micellar concentration of the cholesteryl-based DPA is around $39 \mu \mathrm{M}$. Accordingly, we postulated that the 
cholesteryl moieties of C-DPA might form a barrier preventing effective interactions of the sensitizer with the core DPA of the acceptor (Figures 3A, 3B, and 3C). Results for the S-V quenching studies with C2-DPA are described in the Supporting information (Figure S12); with this acceptor, it was deemed unnecessary to carry out subsequent investigation with $\mathbf{C}_{2}-\mathbf{D P A}$, as no TTA-UC was detected using both QDM and PdTPP (Figures 3D).
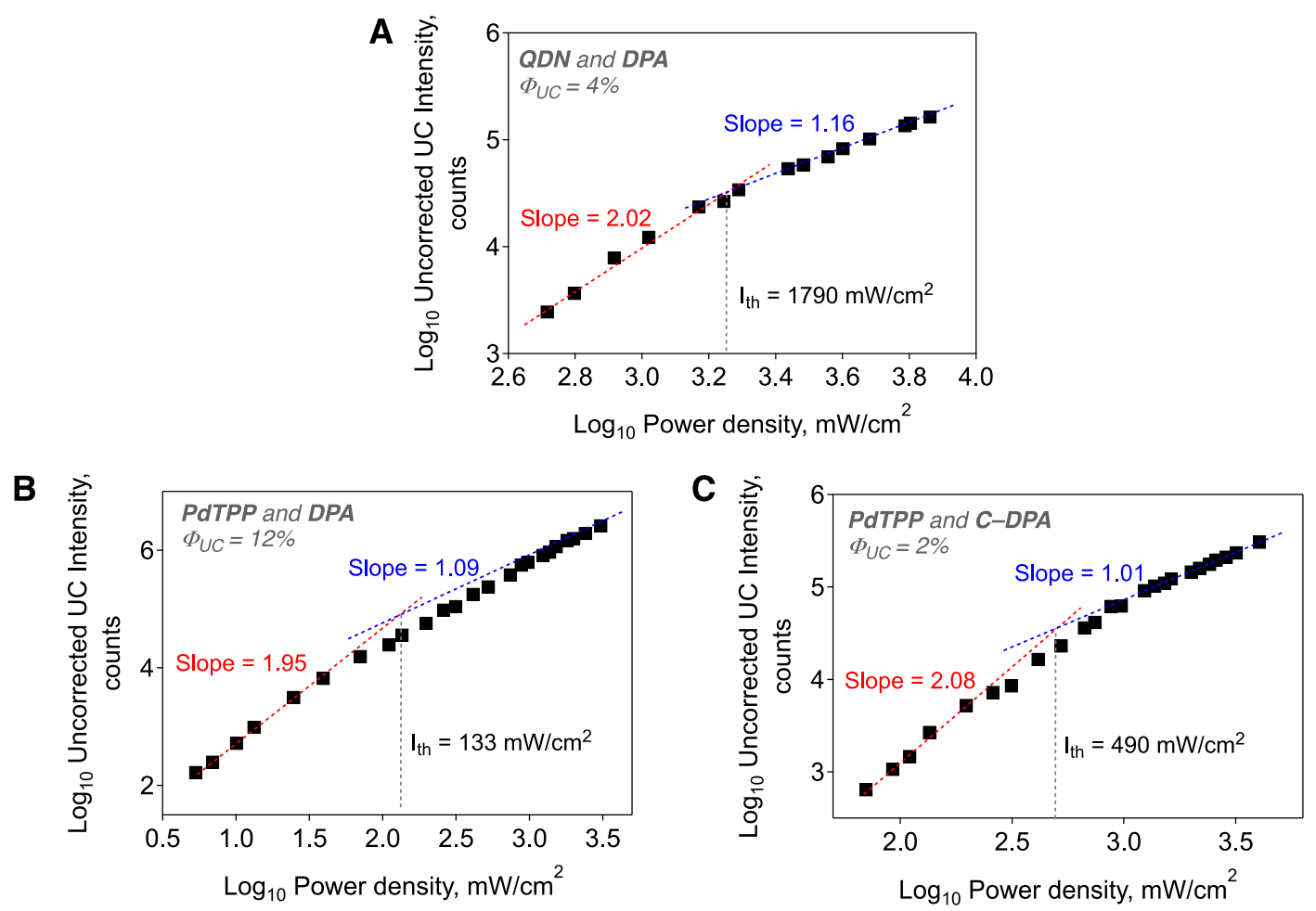

Figure 4. Double logarithmic plot of the upconverted emission for (A) QDN and DPA (B) PdTPP and DPA (C) PdTPP and C-DPA as a function of power density of the $532 \mathrm{~nm} \mathrm{Nd:YAG} \mathrm{laser.}$

Figure 4 shows the double logarithmic plots of the upconverted emission intensity with respect to the $532 \mathrm{~nm}$ excitation power density. All three donor-acceptor pairs display the typical low-to-high power density regimes transition: from quadratic (slope $=2$ ) to linear $($ slope $=1$ ) 
intensity dependence, which is a clear indication of the expected TTA process. ${ }^{13}$ The power density threshold $\left(\mathrm{I}_{\mathrm{th}}\right)^{23}$, where the intensity of the TTA process becomes the dominant deactivation channel for an annihilator triplet state, and the quantum yield of the upconversion process is equal to half of its maximum. From Figure 4, we found that $\mathrm{I}_{\mathrm{th}}$ are 1790,133 , and $490 \mathrm{~mW} / \mathrm{cm}^{2}$ for QDN/DPA, PdTPP/DPA, and PdTPP/C-DPA, respectively. We also hypothesize that the intrinsic radiative and non-radiative decays of the sensitizer could be predominant at low power densities. Under this consideration, at power densities above the $\mathrm{I}_{\mathrm{t}}$, the TTA process was prevalently approaching a maximum delayed fluorescence driven by the TTA-UC process. Also, the high $\mathrm{I}_{\text {th }}$ for QDN/DPA suggests a less efficient (endothermic) energy $\operatorname{transfer}^{14}$ as revealed earlier from the S-V quenching results. For the PdTPP/DPA pair, the threshold intensity is substantially lower than that of QDN/DPA. This observation/result is also in good agreement with the relatively high $k_{T E T}$ from the S-V quenching study (Figure 2A and 2B).

Furthermore, we measured the quantum yield of TTA-UC $\left(\Phi_{U C}\right)$ of the three pairs of donor-acceptor using equation 4 and rhodamine $6 \mathrm{G}^{24}$ as photon counter standard.

$Q Y_{U C}=Q Y_{s t d} \times\left(\frac{A_{s t d}}{A_{U C}}\right) \times\left(\frac{I_{U C}}{I_{s t d}}\right) \times\left(\frac{\eta_{U C}}{\eta_{s t d}}\right)^{2}$

Where $Q Y$ represents the quantum yield, $A$ is absorbance (O.D.) at the excitation wavelength. $I$ denotes the integrated photoluminescence, and $\eta$ is the refractive index of the solvent. std stands for the standard (here, rhodamine 6G ) sample, and $U C$ denotes the upconversion sample. We then estimated that the $\Phi_{U C}$ for (QDN/DPA) is $2 \%$ (4\% after correcting for inner filter effect), 6\% (12\%) for (PdTPP/DPA), and 1\% (2\%) for (PdTPP/C-DPA). 


\section{CONCLUSIONS:}

In summary, this study aims at providing additional molecular tools to the upconversion community wherein over-the-counter annihilators, such as DPA, have been functionalized with cholesteryl tag(s) to afford $\mathbf{C}$-DPA and $\mathbf{C}_{2}$-DPA. In the present work, we successfully employed both DPA and C-DPA to achieve TTA-UC in biorelevant hydrophilic and viscous polymeric liquid PEG200 media. Moreover, this study offers insight on the behavior of these modified annihilators in terms of donor $\bullet \cdot a c c e p t o r$ interactions and the resulting effect on the upconversion process. Using Stern-Volmer quenching experiments, we found that the rate of $\mathbf{P d T P P} \rightarrow \mathbf{C}-\mathbf{D P A}$ TET is three times lower than that of $\mathbf{P d T P P} \rightarrow \mathbf{D P A}$. This reduce $k_{T E T}$ value was hypothesized to originate from the shielding effect of the cholesteryl unit. Beyond a certain concentration threshold, C-DPA formed a self-assembled sub-domains in the PEG200 medium, which was observed by a plateau in the TET efficiency. This finding was further consolidated by investigating the upconversion efficiency of C-DPA upon increasing its concentration. While the cholesteryl units may be beneficial in terms of incorporating dye molecules into lipid bilayers, micelles, and hydrogels and also provide some shielding to molecular oxygen to perform TTA-UC in biological media, we discovered that this substituent may hamper effective donor $\bullet$ acceptor interactions due to reduced molecular mobility and formation of hydrophobic sub-domains that are inaccessible to the triplet energy donor. This is effect is also manifested by the absence of TTA-UC using $\mathbf{C}_{2}-$ DPA as the acceptor. Nevertheless, we successfully achieved TTA-UC with three pairs of donor/acceptor systems QDN/DPA, PdTPP/DPA, and PdTPP/C-DPA with $\Phi_{U C}$ values of 2\% (4\%), $6 \%(12 \%)$, and $1 \%(2 \%)$, respectively. The present study brings new insight into molecular engineering and function of modified biorelevant dyes in order to achieve TTA-UC in biological environments. 


\section{ASSOCIATED CONTENT:}

Data Availability: The data that support the findings of this study are available from the corresponding author upon reasonable request. In addition, details of the synthetic procedures for all precursor of C-DPA and $\mathbf{C}_{2}$-DPA: ${ }^{1} \mathrm{H}$ and ${ }^{13} \mathrm{C}$ NMR spectra, additional UV-vis absorption spectra, emission spectra, this material is available free of charge via the Internet at http://aip.org.

\section{Funding Sources}

National Science Foundation under a CAREER grant no. 1753012 Awarded to AJA.

The Academy of Finland (Grant No. 316893)

Illinois Tech Graduate Kilpatrick and Starr Fieldhouse Fellowships to YJY.

\section{Notes}

The authors declare no competing financial interests.

\section{Authors Contribution}

\# These authors have contributed equally to this work

\section{Corresponding Author}

* aayitou@ iit.edu

\section{ACKNOWLEDGMENT:}

YJY is thankful for the support from the Kilpatrick Graduate Fellowship and the Starr Fieldhouse Research Fellowship Programs at Illinois Tech. The authors thank the National Science Foundation for the generous support to this work under a CAREER grant $\mathrm{N}^{\circ} 1753012$ Awarded 
to AJA. JI, ND and TL also thank the Academy of Finland for the generous support to this work under grant $\mathrm{N}^{\circ} 316893$. 


\section{REFERENCE:}

(1) Zhang, J.; Kouno, H.; Yanai, N.; Eguchi, D.; Nakagawa, T.; Kimizuka, N.; Teranishi, T.; Sakamoto, M. Number of Surface-Attached Acceptors on a Quantum Dot Impacts Energy Transfer and Photon Upconversion Efficiencies. ACS Photonics 2020, 7 (7), 1876-1884.

(2) Nishimura, N.; Gray, V.; Allardice, J. R.; Zhang, Z.; Pershin, A.; Beljonne, D.; Rao, A. Photon Upconversion From Near-Infrared to Blue Light with TIPS-Anthracene as an Efficient Triplet-Triplet Annihilator. ACS Materials Lett. 2019, 1 (6), 660-664.

(3) Zhou, Y.; Castellano, F. N.; Schmidt, T. W.; Hanson, K. On the Quantum Yield of Photon Upconversion via Triplet-Triplet Annihilation. ACS Energy Lett. 2020, 5 (7), 2322-2326.

(4) Cheng, Y. Y.; Nattestad, A.; Schulze, T. F.; MacQueen, R. W.; Fückel, B.; Lips, K.; Wallace, G. G.; Khoury, T.; Crossley, M. J.; Schmidt, T. W. Increased Upconversion Performance for Thin Film Solar Cells: a Trimolecular Composition. Chem. Sci. 2016, 7 (1), 559-568.

(5) Ogawa, T.; Hosoyamada, M.; Yurash, B.; Nguyen, T.-Q.; Yanai, N.; Kimizuka, N. DonorAcceptor-Collector Ternary Crystalline Films for Efficient Solid-State Photon Upconversion. J. Am. Chem. Soc. 2018, 140 (28), 8788-8796.

(6) Wohnhaas, C.; Mailänder, V.; Dröge, M.; Filatov, M. A.; Busko, D.; Avlasevich, Y.; Baluschev, S.; Miteva, T.; Landfester, K.; Turshatov, A. Triplet-Triplet Annihilation Upconversion Based Nanocapsules for Bioimaging Under Excitation by Red and Deep-Red Light. Macromol. Biosci. 2013, 13 (10), 1422-1430.

(7) Gray, V.; Dzebo, D.; Abrahamsson, M.; Albinsson, B.; Moth-Poulsen, K. Triplet-Triplet Annihilation Photon-Upconversion: Towards Solar Energy Applications. Phys Chem Chem Phys 2014, 16 (22), 10345-10352. 
(8) Parker, C. A. Delayed Fluorescence From Naphthalene Solutions. Spectr. Acta 1963, 19 (6), 989-994.

(9) Parker, C. A.; Hatchard, C. G.; Joyce, T. A. P-Type Delayed Fluorescence From Ionic Species and Aromatic Hydrocarbons. J. Mol. Spec. 1964, 14 (1-4), 311-319.

(10) Jin, G.-Q.; Ning, Y.; Geng, J.-X.; Jiang, Z.-F.; Wang, Y.; Zhang, J.-L. Joining the Journey to Near Infrared (NIR) Imaging: the Emerging Role of Lanthanides in the Designing of Molecular Probes. Inorganic Chemistry Frontiers 2020, 7 (2), 289-299.

(11) Ravetz, B. D.; Pun, A. B.; Churchill, E. M.; Congreve, D. N.; Rovis, T.; Campos, L. M. Photoredox Catalysis Using Infrared Light via Triplet Fusion Upconversion. Nature 2019, $565,343-346$.

(12) Jewell, M. P.; Greer, M. D.; Dailey, A. L.; Cash, K. J. Triplet-Triplet Annihilation Upconversion Based Nanosensors for Fluorescence Detection of Potassium. ACS Sens. 2020, $5(2), 474-480$.

(13) Durandin, N. A.; Isokuortti, J.; Efimov, A.; Vuorimaa-Laukkanen, E.; Tkachenko, N. V.; Laaksonen, T. Efficient Photon Upconversion at Remarkably Low Annihilator Concentrations in a Liquid Polymer Matrix: When Less Is More. Chem. Commun. 2018, 54, $14029-14032$.

(14) Isokuortti, J.; Allu, S. R.; Efimov, A.; Vuorimaa-Laukkanen, E.; Tkachenko, N. V.; Vinogradov, S. A.; Laaksonen, T.; Durandin, N. A. Endothermic and Exothermic Energy Transfer Made Equally Efficient for Triplet-Triplet Annihilation Upconversion. J. Phys. Chem. Lett. 2020, 11 (1), 318-324. 
(15) Mongin, C.; Golden, J. H.; Castellano, F. N. Liquid PEG Polymers Containing Antioxidants: a Versatile Platform for Studying Oxygen-Sensitive Photochemical Processes. ACS Appl. Mater. Interfaces 2016, 8 (36), 24038-24048.

(16) Durandin, N. A.; Isokuortti, J.; Efimov, A.; Vuorimaa-Laukkanen, E.; Tkachenko, N. V.; Laaksonen, T. Critical Sensitizer Quality Attributes for Efficient Triplet-Triplet Annihilation Upconversion with Low Power Density Thresholds. J. Phys. Chem. C 2019, 123 (37), 2286522872.

(17) Shokri, S.; Li, J.; Manna, M. K.; Wiederrecht, G. P.; Gosztola, D. J.; Ugrinov, A.; Jockusch, S.; Rogachev, A. Y.; Ayitou, A. J.-L. A Naphtho- P-Quinodimethane Exhibiting Baird's (Anti)Aromaticity, Broken Symmetry, and Attractive Photoluminescence. J. Org. Chem. 2017, 82 (19), 10167-10173.

(18) Shokri, S.; Wiederrecht, G. P.; Gosztola, D. J.; Ayitou, A. J.-L. Photon Upconversion Using Baird-Type (Anti)Aromatic Quinoidal Naphthalene Derivative as a Sensitizer. J. Phys. Chem. C 2017, 121 (42), 23377-23382.

(19) Yun, Y. J.; Kamatham, N.; Manna, M. K.; Li, J.; Liu, S.; Wiederrecht, G. P.; Gosztola, D. J.; Diroll, B. T.; Rogachev, A. Y.; Ayitou, A. J.-L. Interplay Between Energy and Charge Transfers in a Polyaromatic Triplet Donor-Acceptor Dyad. J. Phys. Chem. C 2020, 124 (23), $12205-12212$.

(20) Liu, S.; Wang, X.; Liu, H.; Shen, L.; Zhao, D.; Li, X. Enhancing Triplet Sensitization Ability of Donor-Acceptor Dyads Viaintramolecular Triplet Energy Transfer. J. Mater. Chem. C 2020, 8 (10), 3536-3544. 
(21) Finikova, O. S.; Chen, P.; Ou, Z.; Kadish, K. M.; Vinogradov, S. A. Dynamic Quenching of Porphyrin Triplet States by Two-Photon Absorbing Dyes: Towards Two-Photon-Enhanced Oxygen Nanosensors. J. Photochem. Photobio. A 2008, 198 (1), 75-84.

(22) Geddes, C. D. Optical Halide Sensing Using Fluorescence Quenching: Theory, Simulations and Applications - a Review. Meas. Sci. Technol. 2001, 12 (9), R53-R88.

(23) Monguzzi, A.; Tubino, R.; Meinardi, F. Upconversion-Induced Delayed Fluorescence in Multicomponent Organic Systems: Role of Dexter Energy Transfer. Phys. Rev. B 2008, 77 (15), 155122-155124.

(24) Fischer, M.; Georges, J. Fluorescence Quantum Yield of Rhodamine 6G in Ethanol as a Function of Concentration Using Thermal Lens Spectrometry. Chem. Phys. Lett. 1996, 260 (1-2), 115-118. 
The authors declare no competing financial interests.

Conflict of Interest

The authors declare no competing financial interests.

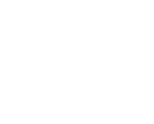

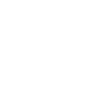

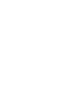

.
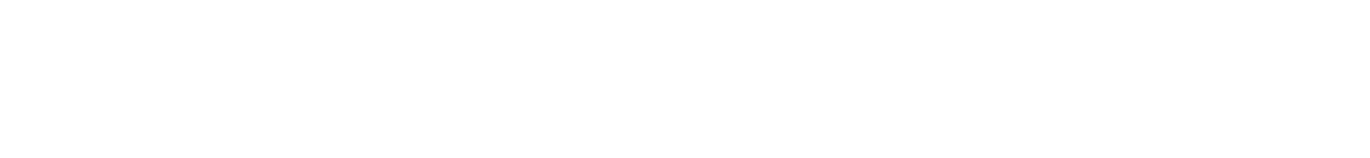

$\sqrt{2}$

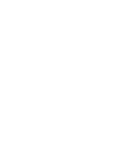

